\title{
ASYMPTOTIC BEHAVIOR OF PERIODIC, PERIODIC BIHARMONIC AND PERIODIC HARMONIC FUNCTIONS*
}

BY

\author{
KENNETH B. HOWELL
}

University of Alabama in Huntsville

\begin{abstract}
The behavior of periodic functions defined on domains containing the upper half space, $\left\{\left(x^{1}, x^{2}, \ldots, x^{n}\right): x^{n}>0\right\}$, is investigated as $x^{n}$ approaches infinity. Bounds on some of the first order derivatives of these functions are obtained which are directly proportional to bounds on derivatives of arbitrary orders in certain directions. It is shown that a periodic biharmonic and a periodic harmonic function can be approximated, respectively, by a third degree and a first degree polynomial in the variable $x^{n}$ and that, as $x^{n}$ approaches infinity, the error in using this approximation vanishes faster than the reciprocal of $x^{n}$ raised to any power.
\end{abstract}

1. Introduction. When dealing with problems on infinite domains, one must have some concern about the behavior of the functions as one "approaches infinity." Often it is desired - and assumed - that the functions rapidly and uniformly approach well-defined limits "at infinity." Unfortunately, desire and assumption are seldom sufficient when coping with reality. Tools must be developed for determining the extent to which expectations can be satisfied. The Laurent series, for example, is a valuable tool when discussing the asymptotic behavior of analytic functions on exterior subdomains of the complex plane. Similar representations are also known to hold for harmonic and biharmonic functions on two- and three-dimensional exterior domains, and have been used by such researchers as Muskhelishvili [5] and Gurtin and Sternberg [1] in studying certain classes of problems in elasticity. It is less clear, however, that the asymptotic behavior one might desire or expect can be guaranteed on domains which are not exterior to compact sets. Indeed, in many situations, just determining what behavior should be desired or expected is a significant problem in itself. A major difficulty is the fact that the asymptotic behavior may be strongly dependent on the direction along which "infinity" is approached. This is the case with the functions considered in this paper. These are the functions which are periodic in one or more directions. Three results will be presented.

*Received December 4, 1985. 
The first, Theorem 3.2, directly relates the asymptotic behavior (as one approaches "infinity" along a direction other than a direction of periodicity) of certain first-order derivatives of a general periodic function to the asymptotic behavior of certain higher-order derivatives. Of possibly greater immediate interest is the second result, Theorem 4.1, showing that the asymptotic behavior of a periodic biharmonic function is given by a third-degree polynomial of one variable. That variable is, essentially, the distance to the domain's boundary. Letting $\rho$ denote this variable, the error in using this polynomial instead of the original function decreases as $\rho$ approaches infinity like $O\left(\rho^{-\sigma}\right)$, where $\sigma$ is arbitrary. Finally, an analogous theorem for periodic functions will be discussed briefly in Sec. V.

II. General preliminaries. Throughout this paper, $\Omega$ will denote an open connected submanifold of $n$-dimensional Euclidean space. Where convenient, points in space will be identified with vectors in $\mathbf{R}^{n}$ via the agency of some fixed Cartesian coordinate system. The induced orthonormal frame of vectors will be denoted by $\left\{\mathbf{e}^{1}, \mathbf{e}^{2}, \ldots, \mathbf{e}^{n}\right\}$ and the corresponding components of any given point in space will be denoted by $\left(x^{1}, x^{2}, \ldots, x^{n}\right)$. $\mathscr{H}$ will denote the "upper half space," $\left\{\left(x^{1}, x^{2}, \ldots, x^{n}\right): x^{n}>0\right\}$. If $\phi$ is a scalar field on $\Omega$ then $\Delta \phi$ denotes the Laplacian of $\phi, \nabla^{k} \phi$ denotes $k$ successive applications of the gradient operator to $\phi$, and $\phi_{, i}$ denotes the $i^{\text {th }}$ component of $\nabla \phi$. As a matter of convenience, only scalar-valued functions will be discussed in this paper. It should be pointed out, however, that the results hold equally well for vector- and tensor-valued functions. Also for convenience, the phrase " $\phi$ is $n$-dimensional" will be adopted as shorthand for "the domain of $\phi$ is an $n$-dimensional manifold." The reader is reminded that $\phi$ is harmonic on $\Omega$ if $\phi$ is in $C^{2}(\Omega)$ and satisfies

$$
\Delta \phi=0
$$

on $\Omega$. The function is biharmonic on $\Omega$ if $\phi$ is in $C^{4}(\Omega)$ and satisfies

$$
\Delta \Delta \phi=0
$$

on $\Omega$. It is a well-known fact that if $\phi$ is harmonic or biharmonic on $\Omega$, then, in fact, $\phi$ is analytic on $\Omega$.

A function, $\phi$, with domain $\Omega$ will be referred to as " $m$-tuply periodic" (or, more simply, as "periodic") if there is a linearly independent set of $m$ vectors, $\left\{\mathbf{p}^{1}, \mathbf{p}^{2}, \ldots, \mathbf{p}^{m}\right\}$, called the periods of $\phi$, such that both of the following hold for each $\mathbf{p}^{i}$ :

1. $\mathbf{x}$ is in $\Omega$ if and only if $\mathbf{x}+\mathbf{p}^{i}$ is in $\Omega$.

2. For each $\mathbf{x}$ in $\Omega, \phi\left(\mathbf{x}+\mathbf{p}^{i}\right)=\phi(\mathbf{x})$.

If, in the above, $m$ equals one, then $\phi$ is "singly periodic." If $m$ equals the dimension of the ambient space, then $\phi$ is "maximally periodic." Finally, if $m$ is one less than the dimension of the ambient space, then $\phi$ will be classified as "almost maximally periodic" (note: if $\Omega$ is an open subset of two-space, then "singly periodic" and "almost maximally periodic" are equivalent, while if $\Omega$ is an open subset of three-space, then "almost maximally periodic" is equivalent to "doubly periodic").

The statements and proofs of the results in this paper will be simplified by the adoption of two conventions. The first is that given any $m$-tuply periodic function, $\phi$, then $\left\{\mathbf{p}^{1}, \mathbf{p}^{2}, \ldots, \mathbf{p}^{m}\right\}$ is a fixed linearly independent set of periods for $\phi$ and, unless otherwise 
stated, only elements of this fixed set will be termed "periods of $\phi$. " The second convention is that, given an $m$-tuply periodic function, $\phi$, then the Cartesian coordinate system mentioned in the first paragraph of this section is chosen so that

$$
\begin{aligned}
\mathbf{p}^{1} & =p_{1}^{1} \mathbf{e}^{1}, \\
\mathbf{p}^{2} & =p_{1}^{2} \mathbf{e}^{1}+p_{2}^{2} \mathbf{e}^{2}, \\
& \vdots \\
\mathbf{p}^{k} & =\sum_{i=1}^{k} p_{i}^{k} \mathbf{e}^{i} \quad \text { for } i=1,2, \ldots, m .
\end{aligned}
$$

Such a choice is, of course, always possible.

III. General periodic functions. Throughout this section, $\phi$ denotes an $m$-tuply periodic function with periods $\mathbf{p}^{1}, \mathbf{p}^{2}, \ldots, \mathbf{p}^{m}$. Its domain $\Omega$ will be said to be "boundedly periodically connected" if there is a fixed finite constant (denoted, henceforth, by $M$ ) such that given any $\mathbf{x}$ in $\Omega$ and any period, $\mathbf{p}^{i}$, then there is a smooth path (henceforth denoted by $l^{i}$ ) in $\Omega$ from $\mathbf{x}$ to $\mathbf{x}+\mathbf{p}^{i}$ of length less than or equal to $M$. The domain, $\Omega$, will be called "directly periodically connected" if it is boundedly periodically connected and if, in addition, each $l^{i}$ may be chosen so that every tangent to $l^{i}$ is in the span of $\left\{\mathbf{p}^{1}, \mathbf{p}^{2}, \ldots, \mathbf{p}^{m}\right\}$.

Lemma 3.1. If $\phi$ is in $C^{2}(\Omega)$ and $\Omega$ is boundedly periodically connected, then, for any $\mathbf{p}^{i}$ and any $\mathbf{x}$ in $\Omega$,

$$
\left|\nabla \phi(\mathbf{x}) \cdot \mathbf{p}^{i}\right| \leqslant \frac{1}{2} M^{2} \sup \left\{\|\nabla \nabla \phi(\overline{\mathbf{x}})\|: \overline{\mathbf{x}} \in l_{x}^{i}\right\} .
$$

Proof. Using the periodicity of $\phi$ and basic calculus:

$$
\begin{aligned}
\nabla \phi(\mathbf{x}) \cdot \mathbf{p}^{i} & =\nabla \phi(\mathbf{x}) \cdot \mathbf{p}^{i}-\left[\phi\left(\mathbf{x}+\mathbf{p}^{i}\right)-\phi(\mathbf{x})\right] \\
& =\int_{\mathbf{y}=\mathbf{x}}^{\mathbf{x}+\mathbf{p}^{i}}[\nabla \phi(\mathbf{x})-\nabla \phi(\mathbf{y})] \cdot d \mathbf{y} \\
& =-\int_{\mathbf{y}=\mathbf{x}}^{\mathbf{x}+\mathbf{p}^{j}}\left[\int_{\mathbf{z}=\mathbf{x}}^{\mathbf{y}} \nabla \nabla \phi(\mathbf{z}) d \mathbf{z}\right] \cdot d \mathbf{y},
\end{aligned}
$$

where the integration is carried out over the path $l^{i}$. Computation of the obvious upper bound for the last iterated integral in (3.2) leads directly to (3.1.)

The bound derived above is rather crude and can be improved in many cases of interest. For example, let $\Omega$ be directly periodically connected, and let $\overline{\mathbf{x}}(s)$ be the arc length parameterization of $l^{i}, \mathbf{t}(s)$ the corresponding unit tangent, and $N$ the length of $l^{i}$. Then (3.2) implies the following:

$$
\begin{aligned}
\left|\nabla \phi(\mathbf{x}) \cdot \mathbf{p}^{i}\right| & =\left|\int_{s=0}^{N} \int_{r=0}^{s}[\nabla \nabla \phi(\overline{\mathbf{x}}(r)) \mathbf{t}(r)] \cdot \mathbf{t}(s) d r d s\right| \\
& \leqslant \frac{1}{2} N^{2} \sup \left\{|\nabla \nabla \phi(\overline{\mathbf{x}}) \cdot(\mathbf{n} \otimes \mathbf{m})|: \overline{\mathbf{x}} \in l_{\mathbf{x}}^{i} ; \mathbf{n}, \mathbf{m} \in \mathscr{S}^{m}\right\} \\
& \leqslant \frac{1}{2} M^{2} \sup \left\{|\nabla \nabla \phi(\overline{\mathbf{x}}) \cdot(\mathbf{n} \otimes \mathbf{m})|: \overline{\mathbf{x}} \in l_{\mathbf{x}}^{i} ; \mathbf{n}, \mathbf{m} \in \mathscr{S}^{m}\right\},
\end{aligned}
$$


where $\mathscr{S}^{m}$ is the unit $m$-sphere in the span of the $\mathbf{p}^{i}$ 's. In particular, if $l^{i}$ may be taken to be a straight line path from $\mathbf{x}$ to $\mathbf{x}+\mathbf{p}^{i}$, then (3.3) may be further refined to

$$
\left|\nabla \phi(\mathbf{x}) \cdot \mathbf{p}^{i}\right| \leqslant \frac{1}{2} \sup \left\{\left|\nabla \nabla \phi(\overline{\mathbf{x}}) \cdot\left(\mathbf{p}^{i} \otimes \mathbf{p}^{i}\right)\right|: \overline{\mathbf{x}} \in l_{\mathbf{x}}^{i}\right\} .
$$

Some observations are in order here. The first is that, by the choice of coordinate system made in the previous section, each $\mathbf{e}^{i}$ with $i \leqslant m$ may be expressed as a linear combination of the $\mathbf{p}^{i}$ 's, say

$$
\mathbf{e}^{i}=\sum_{j=1}^{m} e_{j}^{i} \mathbf{p}^{j}
$$

so for each $i=1,2, \ldots, m$, if $\Omega$ is directly periodically connected, then

$$
\begin{aligned}
\left|\phi_{. i}(\mathbf{x})\right| & =\left|\nabla \phi(\mathbf{x}) \cdot \mathbf{e}^{i}\right| \\
& \leqslant \sum_{j=1}^{m}\left|e_{j}^{i}\right|\left|\nabla \phi(\mathbf{x}) \cdot \mathbf{p}^{j}\right| \\
& \leqslant \sum_{j=1}^{m}\left|e_{j}^{i}\right| \frac{1}{2} M^{2} K(\mathbf{x}),
\end{aligned}
$$

where

$$
K(\mathbf{x})=\sup \left\{|\nabla \nabla(\overline{\mathbf{x}}) \cdot(\mathbf{n} \otimes \mathbf{m})|: \overline{\mathbf{x}} \in l_{\mathbf{x}}^{i} ; \mathbf{n}, \mathbf{m} \in \mathscr{S}^{m} ; i=1,2, \ldots, m\right\} .
$$

But, computing $K(\mathbf{x})$,

$$
\begin{aligned}
K(\mathbf{x}) & =\sup \left\{\left|\sum_{j=1}^{m} \Sigma_{k=1}^{m} \phi_{, j k}(\overline{\mathbf{x}}) n_{j} m_{k}\right|: \overline{\mathbf{x}} \in l_{\mathbf{x}}^{i} ; i=1,2, \ldots, m ; \sum_{j=1}^{m}\left(n_{j}\right)^{2}=\sum_{k=1}^{m}\left(m_{k}\right)^{2}=1\right\} \\
& \leqslant \sup \left\{\sum_{j=1}^{m} \Sigma_{k=1}^{m}\left|\phi_{, j k}(\overline{\mathbf{x}})\right|: \overline{\mathbf{x}} \in l_{\mathbf{x}}^{i} ; i=1,2, \ldots, m\right\} \\
& \leqslant m^{2} \sup \left\{\left|\phi_{, j k}(\overline{\mathbf{x}})\right|: \overline{\mathbf{x}} \in l_{\mathbf{x}}^{i} ; i, j, k=1,2, \ldots, m\right\} .
\end{aligned}
$$

Combining (3.4) with (3.5), it can be seen that there is a fixed finite constant, $c$, dependent only on the $\mathbf{p}^{i}$ 's, such that, for each $i=1,2, \ldots, m$,

$$
\left|\phi_{, i}(\mathbf{x})\right| \leqslant c \sup \left\{\left|\phi_{, j k}(\overline{\mathbf{x}})\right|: \overline{\mathbf{x}} \in l_{\mathbf{x}}^{l} ; j, k, l=1,2, \ldots, m\right\} .
$$

But, if $\phi$ is periodic, then so is each $\phi_{. i}$, and the above arguments can be repeated to obtain bounds on $\phi_{, i}$ in terms of bounds on the $\phi_{, i j k}$ 's. More precisely, for each $i=1,2, \ldots, m$ and $\mathbf{x}$ in $\Omega$, one has

$$
\left|\phi_{, i}(\mathbf{x})\right| \leqslant c^{2} \sup \left\{\left|\phi_{, \alpha \beta \gamma}(\hat{\mathbf{x}})\right|: \hat{\mathbf{x}} \in l_{\hat{\mathbf{x}}}^{j} ; \overline{\mathbf{x}} \in l_{\mathbf{x}}^{k} ; j, k, \alpha, \beta, \gamma=1,2, \ldots, m\right\} .
$$

The direction in which this discussion is leading should now be apparent. It, combined with a suitable appeal to the god of induction, constitutes the proof of the following theorem.

THEOREM 3.2. Let $\phi$ be periodic and have a directly periodically connected domain, $\Omega$. Then there exists a constant, $c$, dependent only on $\left\{\mathbf{p}^{1}, \mathbf{p}^{2}, \ldots, \mathbf{p}^{m}\right\}$, such that, for any given $i=1,2, \ldots, m$ and any $k$ for which $\phi$ is in $C^{k}(\Omega)$, the following bound holds for all $\mathbf{x}$ in $\Omega$ :

$$
\left|\phi_{, i}(\mathbf{x})\right| \leqslant c^{k-1} \sup \left\{\left|\phi_{, \alpha_{1}, \alpha_{2}, \ldots, \alpha_{k}}(\overline{\mathbf{x}})\right|: \overline{\mathbf{x}} \in \mathscr{V}_{\mathbf{x}}^{k} ; \alpha_{j}=1,2, \ldots, m\right\},
$$


where

$$
\mathscr{V}_{\mathbf{x}}=\mathbf{x}+\operatorname{span}\left\{\mathbf{p}^{1}, \mathbf{p}^{2}, \ldots, \mathbf{p}^{m}\right\}
$$

and

$$
\mathscr{V}_{\mathbf{x}}^{k}=\left\{\overline{\mathbf{x}}: \overline{\mathbf{x}} \in \mathscr{V}_{\mathbf{x}} \text { and }|\mathbf{x}-\overline{\mathbf{x}}|<k M\right\}
$$

The following corollary follows immediately from Theorem 3.2. It will be used in the next section.

Corollary 3.3. Let $\phi$ be as in the previous theorem. Let $\Sigma$ be some fixed closed set of points in space and for each point, $\mathbf{x}$, define $\rho=\rho(\mathbf{x})$ to be the distance from $\mathbf{x}$ to $\Sigma$. Suppose, for some integer, $k$, greater than 2 and some arbitrary real constant, $\sigma$, that $\phi$ is in $C^{k}(\Omega)$ and

$$
\nabla^{k} \phi(\mathbf{x})=O\left(\rho^{\sigma}\right) \quad \text { as } \rho=\rho(\mathbf{x}) \rightarrow \infty \text { on } \Omega .
$$

Then, for each $i=1,2, \ldots, m$,

$$
\phi_{, i}(\mathbf{x})=O\left(\rho^{\sigma}\right) \quad \text { as } \rho=\rho(\mathbf{x}) \rightarrow \infty \text { on } \Omega .
$$

IV. Periodic biharmonic functions. The primary goal of this section is to prove the following theorem.

THEOREM 4.1. Let $\phi$ be a two- or three-dimensional, almost maximally periodic, biharmonic function whose domain contains the upper half "space", $\mathscr{H}$. For each point, x, let $\rho=\rho(\mathbf{x})$ denote the distance between $\mathbf{x}$ and the boundary of $\mathscr{H}$. Assume that for some real $\beta$

$$
\phi(\mathbf{x})=O\left(\rho^{\beta}\right) \quad \text { as } \rho=\rho(\mathbf{x}) \rightarrow \infty \text { on } \mathscr{H} .
$$

Then there exists a third-degree polynomial of one variable, $I$, such that for any positive real constant, $\sigma$,

$$
\phi(\mathbf{x})-I(\rho)=O\left(\rho^{-\sigma}\right) \quad \text { as } \rho=\rho(\mathbf{x}) \rightarrow \infty \text { on } \mathscr{H} .
$$

The next three lemmas all play a significant role in the proof of Theorem 4.1. Of these lemmas, only the third need be proved here. The first is easily recognized as a special case of a more general theorem proven in [2] (Theorem 4.3 of that paper), while the second lemma is a rather straightforward corollary of the first. In each lemma, $\rho=\rho(\mathbf{x})$ is as defined above in Theorem 4.1.

LEMMA 4.2. Let $\phi$ be a two- or three-dimensional biharmonic function on $\mathscr{H}$ and assume there exist a positive-valued, locally integrable, nonincreasing function, $f$, on $[0, \infty)$ and two real constants, $c$ and $\beta$, with $c$ positive, such that for each $\mathbf{x}$ in $\mathscr{H}$

$$
|\phi(\mathbf{x})|<c \rho^{\beta} f(\rho) \text {. }
$$

Then for each $\mathbf{x}$ in $\mathscr{H}$

$$
|\nabla \phi(\mathbf{x})|<B c \rho^{\beta-1}(a \rho)
$$

where

$$
a=2^{-3 / 2}, \quad B=9 \sqrt{2}[2 \sqrt{2}]^{|\beta|} .
$$


Lemma 4.3. Let $\phi$ be a two- or three-dimensional biharmonic function on $\mathscr{H}$ and assume there are a positive-valued, locally integrable, nonincreasing function, $f$, on $[0, \infty)$ and two real constants, $c$ and $\beta$, with $c$ positive, such that for each $\mathbf{x}$ in $\mathscr{H}$

$$
|\phi(\mathbf{x})|<c \rho^{\beta} f(\rho) .
$$

Then for each $\mathbf{x}$ in $\mathscr{H}$ and each $k=1,2, \ldots$ :

$$
\left|\nabla^{k} \phi(\mathbf{x})\right|<B_{k} c \rho^{\beta-k} f\left(a^{k} \rho\right),
$$

where

$$
B_{k}=(9 \sqrt{2})^{k}(2 \sqrt{2})^{s}
$$

and

$$
s=|\beta|+|\beta-1|+|\beta-2|+\cdots+|\beta-k+1| .
$$

The final lemma (which will be proved) applies even with three-dimensional, singly periodic, biharmonic functions. The reader is reminded that this lemma is stated assuming the conventions discussed at the end of Sec. II.

Lemma 4.4. Let $\phi$ be a two- or three-dimensional, $m$-tuply periodic, biharmonic function whose domain contains $\mathscr{H}$. Let the coordinate system be chosen so that the periods are contained in the span of the set of $\mathbf{e}^{i}$ s with $i$ no greater than $m$. Assume that for some real constant, $\beta$,

$$
\phi(\mathbf{x})=O\left(\rho^{\beta}\right) \quad \text { as } \rho=\rho(\mathbf{x}) \rightarrow \infty \text { on } \mathscr{H} .
$$

Then given any real constant, $\sigma$, and any $i=1,2, \ldots, m$

$$
\phi_{, i}(\mathbf{x})=O\left(\rho^{-\sigma}\right) \quad \text { as } \rho=\rho(\mathbf{x}) \rightarrow \infty \text { on } \mathscr{H} .
$$

Proof. Without loss of generality it may be assumed that $-\sigma<\beta$ and that $\sigma$ is positive.

Let $\sigma$ be chosen and fix $k$ so that $-\sigma$ is no less than $\beta-k$. By Lemma 4.3, then

$$
\nabla^{k} \phi(\mathbf{x})=O\left(\rho^{-\sigma}\right) \quad \text { as } \rho=\rho(\mathbf{x}) \rightarrow \infty \text { on } \mathscr{H} .
$$

But Corollary 3.3 is now applicable with the boundary of $\mathscr{H}$ used as $\Sigma$. And from that, (4.3) follows immediately.

Final discussion of Theorem 4.1 will be simplified by assuming that $\phi$ is three-dimensional. There is no loss of generality in this since one can, clearly, view any two-dimensional, almost maximally periodic, biharmonic function as a corresponding three-dimensional, almost maximally periodic, biharmonic function in which the derivative in the third direction vanishes. In line with this simplification, components of a point in space, $\mathbf{x}$, with respect to the given coordinate system, will be denoted by $(x, y, z)$ as well as by $\left(x^{1}, x^{2}, x^{3}\right)$. Using this notation, (4.1) and (4.2) can be rewritten, respectively, as

$$
\begin{aligned}
\phi(x, y, z) & =O\left(z^{\beta}\right) \quad \text { as } z \rightarrow \infty, \\
\phi(x, y, z)-I(z) & =O\left(z^{-\sigma}\right) \quad \text { as } z \rightarrow \infty .
\end{aligned}
$$

Proof of Theorem 4.1 (with $m=3$ ). By Lemma 4.4 and the conventions adopted at the end of Sec. II:

$$
\begin{aligned}
& \phi_{, 1}(x, y, z)=O\left(z^{-\sigma}\right) \text { as } z \rightarrow \infty, \\
& \phi_{, 2}(x, y, z)=O\left(z^{-\sigma}\right) \text { as } z \rightarrow \infty,
\end{aligned}
$$


for any real $\sigma$. Applying Lemma 4.3 to the above $\phi_{, i}$ 's yields

$$
\phi_{, i i j j}(x, y, z)=O\left(z^{-\sigma}\right) \quad \text { as } z \rightarrow \infty
$$

for any real $\boldsymbol{\sigma}$, and any possible choice of $i$ and $j$ as long as both are not equal to three. On the other hand, $\phi$ satisfies $\Delta \Delta \phi=0$. Solving this equation for $\phi_{, 3333}$ and employing (4.8) leads to the realization that

$$
\phi_{.3333}(x, y, z)=O\left(z^{-\sigma}\right) \quad \text { as } z \rightarrow \infty
$$

for any real $\sigma$.

Fixing $x$ and $y$ and integrating (4.9) yields the following:

$$
\begin{aligned}
\phi_{, 333}(x, y, \bar{z})-\phi_{.333}(x, y, z) & =\int_{z}^{\bar{z}} \phi_{, 3333}(x, y, t) d t \\
& =O\left(z^{-\sigma}\right) \quad \text { as } z \rightarrow \infty
\end{aligned}
$$

for any $z<\bar{z}$ and any positive $\sigma$. Standard Cauchy sequence arguments then imply the existence of a scalar, $A$, which is independent of $z$, such that for any positive $\sigma$

$$
\phi_{, 333}(x, y, z)-\frac{1}{6} A=O\left(z^{-\sigma}\right) \quad \text { as } z \rightarrow \infty .
$$

Repeating these arguments three or more times reveals the existence of three more scalars, $B, C$, and $D$, such that for any positive $\sigma$

$$
\phi(x, y, z)-\left[A x^{3}+B z^{2}+C z+D\right]=O\left(z^{-\sigma}\right) \quad \text { as } z \rightarrow \infty .
$$

Referring back to (4.6) and (4.7) and the periodicity of $\phi$, it is immediately obvious that these scalars are also independent of $x$ and $y$.

V. Periodic harmonic functions. If a function, $\phi$, is harmonic, then it is automatically biharmonic and Theorem 4.1 applies. Since the Laplacian is a second-order operator, however, the proof of Theorem 4.1 can easily be modified to prove the following theorem.

THEOREM 5.1. Let $\phi$ be a two- or three-dimensional, almost maximally periodic, harmonic function whose domain contains the upper half "space", $\mathscr{H}$. For each point, x, let $\rho=\rho(\mathbf{x})$ denote the distance between $\mathbf{x}$ and the boundary of $\mathscr{H}$. Assume that for some real $\beta$

$$
\phi(\mathbf{x})=O\left(\rho^{\beta}\right) \quad \text { as } \rho=\rho(\mathbf{x}) \rightarrow \infty \text { on } \mathscr{H} .
$$

Then there exists a first-degree polynomial of one variable, $I$, such that for any positive real constant, $\sigma$,

$$
\phi(\mathbf{x})-I(\rho)=O\left(\rho^{-\sigma}\right) \quad \text { as } \rho=\rho(\mathbf{x}) \rightarrow \infty \text { on } \mathscr{H} .
$$

VI. Comments. One area in which the results discussed here can be applied is in the field of elasticity, where many of the solutions are given by biharmonic vector and tensor fields. Indeed, rather restrictive versions of Theorem 4.1 had already been proved for certain periodic strain states in [3] and [4]. Using the more general results from the paper at hand, 
those results can, in turn, be shown to apply to a much more general class of elastic states. Since a complete discussion of this and other implications of Theorem 4.1 to elasticity goes beyond the scope of this paper, it will be reserved for a future paper.

\section{REFERENCES}

[1] M. E. Gurtin and E. Sternberg, Theorems in linear elastostatics for exterior domains, Arch. Rat. Mech. Anal. 8, 99-119 (1961)

[2] K. B. Howell, Directionally dependent asymptotic behavior of biharmonic functions with applications to elasticity, SIAM J. Math. Anal. 16, 822-847 (1985)

[3] K. B. Howell, Asymptotic behavior of periodic strain states, SIAM J. Math. Anal. 17, 197-217 (1986)

[4] K. B. Howell, The asymptotic behavior of doubly periodic strain states, J. Elasticity 16, 43-61 (1986)

[5] N. K. Muskhelishvili, Some basic problems of the mathematical theory of elasticity (translation by J. R. M. Radok), Noordhoff, Groningen (1953) 